\title{
Human Heredity
}

Abel, L. 251

Agapova, R.K. 224

Akanmori, B.D. 382

Aladhami, S.M.S. 146

Alcaïs, A. 251

Al-Khawari, M. 242

Alsaeid, K. 242

Altisent, C. 266

Alvarado Esquivel, C. 263

Ameen, R. 158

Amorim, A. 189

Ankrah, N.-A. 283

Aragaki, C. 194

Arjas, E. 308

Bailey-Wilson, J.E. 126

Ben-Amitai, D. 325

Blanton, S.H. 102

Bogard, C. 247

Boger, S. 370

Bonamico, M. 180

Bonuccelli, G. 331

Brandful, J.A.M. 382

Cardon, L.R. 350

Chapman, N.H. 166

Charlier, N. 304

Chen, B. 268

Clinical Centers of CF French Registry 142

Collins, A. 76

Coskun, T. 102

Costa, X. 320

Cotrina, M. 320

Curtis, D. 133

Dagnino, F. 331

Dean, M. 318

De Buyzere, M. 263

De Crozes, D. 142

Degioanni, A. 142

De Haene, H. 263

Delanghe, J. 263

Demirkol, M. 102

Deybach, J.-C. 247

DiMe Study Group 308

Dorman, J.S. 242

Doukas, C. 237

Dragos, V. 234

Ekuban, F.A. 382

Elston, R.C. 126

Eriksson, A.W. 57
Farssac, E. 266

Feingold, J. 118, 142

Filocamo, M. 331

Franchina, M. 112

Fu, S. 268

Galactéros, F. 118

Gallardo, D. 266

Garinis, G. 237

Ghosh, S. 43

Gilbert, J.R. 151

Glaser, B. 325

Glavač, D. 140,318

Gokgoz, N. 162

Golouh, R. 140

Gordon, D. 175

Gould, C.P. 146

Grillo, R. 180

Guilloud-Bataille, M. 142

Gülden, H. 102

Guo, J. 201

Guo, S.-W. 271, 286

Gyan, B.A. 382

Hahnen, E. 171

Haider, M.Z. 242

Hampe, J. 91

Heimesaat, M. 370

Hodge, S.E. 359

Hoh, J.J. 85, 359

Horev, L. 325

Horowitz, S.H. 166

Hsu, L. 194

Ioannou, P. 151

Isasi, C. 227

Jardi, R. 320

Javaheri, R. 102

Jorde, L.B. 57

Kádasi, L. 171

Kay, P.H. 112

Kayserili, H. 162

Keats, B.J. 126

Kere, J. 57

Keyaerts, E. 304

Khorte, M.V. 224

Kim, J.-j. 365

Kirdar, B. 162

Kollee, L.A. 370

Kolokithopoulos, D. 237
Komel, R. 234

Krolewski, A. 184

Kumar, A. 151

Laird, N. 211

Landa, B.L. 102

Langlois, M. 263

Larrègue, M. 322

Lee, C. 365

Lee, C.-u. 365

Li, C.C. 22

Li, L. 201

Li, P. 268

Li, W. 334

Limborska, S.A. 224

Lin, D. 151

Liovic, M. 234

Lisi, V. 175

Lopez-Talavera, J.C. 320

Majumder, P.P. 43

Mandal, D.M. 126

Mazzilli, M.C. 180

Menounos, P. 237

Metzker, A. 325

Minder, E.I. 247

Miranda, C. 189

Mirandola, S. 175

Miravitlles, M. 320

Montes, F. 257

Mora, B. 180

Morton, N.E. 5

Mottes, M. 175

Mouélé, R. 118

Muhammad, F.A. 146

Nance, W.E. 102

Navas, M.A. 370

Neel, J.V. 14

Newey, S. 151

Ni, X. 201

Nordmann, Y. 247

Norrgard, K.J. 102

Nurbaev, S.D. 224

Nürnberg, P. 91

Nyman, D. 57

Onkamo, P. 308

Ott, J. 4, 85

Ozalp, I. 102

Ozkinay, F. 162

\section{KARGER}

(c) 2000 S. Karger AG, Base

Fax + 41613061234

E-Mail karger@karger.ch

www.karger.com
Accessible online at:

www. karger.com/journals/hhe 
Paik, I.-h. 365

Pambou, O. 118

Pandya, A. 102

Pascual, C. 320

Patrinos, G.P. 237

Peltonen, L. 66

Pericak-Vance, M.A. 151

Petronzelli, F. 180

Pettenati, M.J. 151

Pignatti, P.F. 175

Pitkäniemi, J. 308

Plaza, M. 257

Podrumac, B. 234

Pogoda, T.V. 224

Pomponio, R.J. 102

Potočnik, U. 140

Prata, M.J. 189

Province, M.A. 34

Puig, L. 266

Puy, H. 247

Qiu, H. 151

Quaye, I.K.E. 382

Rabinowitz, D. 184, 211, 227

Rao, D.C. 34

Rao, N. 151

Rault, G. 142

Ravnik-Glavač, M. 140, 318

Regis, S. 331

Reich, J. 334

Rigatelli, F. 175

Rocha, J. 189

Rodriguez-Frias, F. 320

Rogers, M.A. 322
Rosen, D.R. 166

Ruá, E. 257

Rüfenacht, U.B. 247

Saavedra-Matiz, C.A. 166

Savas, S. 162

Schaumberg, D. 184

Schneider-Yin, X. 247

Schreiber, S. 91

Schweizer, J. 322

Shaltout, A. 242

Sham, P.C. 133

Shea, S. 227

Siegmund, K.D. 205

Slominsky, P.A. 224

Spitsyn, V.A. 224

Stoffel, M. 370

Stroppiano, M. 331

Struyf, F. 304

Swango, K.L. 102

Tapadar, P. 43

Tegos, C. 237

Thoelen, I. 304

Todorov, A.A. 205

Toh, K.-y. 365

Tokatli, A. 102

Tuomilehto, J. 308

Tuomilehto-Wolf, E. 308

Usanga, E.A. 158

Vabres, P. 322

Vaisse, C. 370

Van der Donck, I. 304
Van Ranst, M. 304

Vardy, D. 325

Vidal, F. 266

Vidal, R. 320

Vouk, K. 234

Watkins, W.S. 57

Weissbecker, K. 126

Wienker, T. 91

Wijsman, E.M. 166

Wilson, A.F. 126

Winter, H. 322

Wirth, B. 171

Wolf, B. 102

Wuu, J. 304

Xia, J. 201

Xia, X.-J. 102

$\mathrm{Xu}, \mathrm{P} .151$

Yang, Q. 227

$\mathrm{Yu}$, J. 268

Yuksel-Apak, M. 162

Zanchi, A. 184

Zat'ková, A. 171

Zervas, C. 237

Zhang, G. 268

Zhao, J.H. 133

Zhao, L.P. 194

Zlotogorski, A. 325

Zolezzi, F. 175 